\title{
High expression of RACK1 is associated with poor prognosis in patients with pancreatic ductal adenocarcinoma
}

\author{
HAILIN HAN $^{1 *}$, DONGMEI WANG ${ }^{2 *}$, MAOWU YANG ${ }^{2}$ and SHENHAO WANG ${ }^{3}$ \\ Departments of ${ }^{1}$ Radiology and ${ }^{2}$ Gastroenterology, The Second People's Hospital of \\ Liaocheng Affiliated to Taishan Medical College, Liaocheng, Shandong 252600; ${ }^{3}$ Department of Gastroenterology, \\ The Second Affiliated Hospital, School of Medicine, Xi'an Jiaotong University, Xi'an, Shaanxi 710004, P.R. China
}

Received October 31, 2015; Accepted April 13, 2017

DOI: $10.3892 / \mathrm{ol} .2017 .7539$

\begin{abstract}
Receptor for activated C kinase 1 (RACK1) is associated with certain aspects of cancer biology and signaling pathways, but its function in pancreatic ductal adenocarcinoma (PDAC) remains unknown. In the present study, 157 patients with PDAC were enrolled. RACK1 mRNA and protein expression levels were analyzed in PDAC tissues and matched adjacent noncancerous tissues by reverse transcription-quantitative polymerase chain reaction and western blotting. RACK1 expression levels in paraffin-embedded PDAC tissues were determined by immunohistochemistry. The associations between RACK 1 expression and clinical data were evaluated using $\chi^{2}$ analysis. The relationship between RACK1 expression and the survival data of patients was analyzed using Kaplan-Meier and log rank tests. RACK1 mRNA and protein were revealed to be overexpressed in PDAC tumor tissues compared with adjacent noncancerous tissues. RACK1 expression was associated with clinical stage $(\mathrm{P}=0.001)$, lymph node invasion $(\mathrm{P}=0.003)$ and liver metastasis $(\mathrm{P}=0.001)$. Furthermore, patients with PDAC and high RACK1 expression demonstrated shorter overall survival times compared with patients with low RACK1 expression $(\mathrm{P}=0.002)$. Multivariate analysis indicated that RACK1 overexpression was an independent prognostic factor for patients with PDAC. Overexpression
\end{abstract}

Correspondence to: Dr Hailin Han, Department of Radiology, The Second People's Hospital of Liaocheng Affiliated to Taishan Medical College, 306 Jiankang Road, Liaocheng, Shandong 252600, P.R. China

E-mail: hailin.han@yahoo.com

Dr Shenhao Wang, Department of Gastroenterology, The Second Affiliated Hospital, School of Medicine, Xi'an Jiaotong University, 157, Xiwu Road, Xi'an, Shaanxi 710004, P.R. China

E-mail: dr_shwang@sina.com

*Contributed equally

Key words: pancreatic ductal adenocarcinoma, receptor for activated $\mathrm{C}$ kinase 1 , prognosis of RACK1 may contribute to tumor progression, and may be a potential prognostic biomarker for patients with PDAC.

\section{Introduction}

Pancreatic ductal adenocarcinoma (PDAC) is a serious disease with the lowest five-year survival rate among all solid cancers $(<5 \%)$ (1). At present, PDAC is the fourth leading cause of cancer-associated mortality in the USA and its incidence has increased in past decades (2). Poor survival is probably due to early local invasion and metastasis, which means that potentially curative surgery is no longer an option for $85 \%$ of patients at the point of diagnosis (3). Furthermore, patients who have undergone surgery may experience local recurrence or metastasis within 1 year (4). Therefore, an improved understanding of the biological processes underlying PDAC, in particular the aggressive nature of its invasion, is warranted.

Receptor for activated C kinase 1 (RACK1), also known as GNB2L1, is a 36-kilodalton cytosolic protein (5). It was first reported as an anchoring protein with seven WD40 (Trp-Asp) repeats (6). RACK1 has been revealed to interact with multiple signaling molecules, including protein kinase $\mathrm{C}(\mathrm{PKC})$, period circadian clock 1 and $\operatorname{Src}(7,8)$. It is regarded as a platform for various signal transduction pathways. RACK1 is involved in cell division, invasion and migration in cancer $(5,9,10)$. However, the function of RACK1 in pancreatic ductal adenocarcinoma has not yet been investigated.

The aim of the present study was to assess the expression of RACK1 in PDAC tumor tissues and adjacent noncancerous tissues, and to compare RACK1 expression with clinicopathological characteristics. The value of RACK1 as a prognostic biomarker for patients with PDAC was then evaluated.

\section{Materials and methods}

Patients and tissue samples. Archived and formalin-fixed, paraffin-embedded tumor tissues were obtained from 157 patients with PDAC, who were underwent surgery between September 2003 and March 2011 in the Department of Hepatobiliary Surgery, The Second Affiliated Hospital of Xi'an Jiaotong University (Xi'an, China). Informed consent was given by all patients prior to surgery. The group consisted of 76 men and 81 women with an average age of 56 years 
(range, 29-81 years). The clinicopathological characteristics are presented in Table I. The diagnosis of all patients was confirmed by pathologists. The histological classification and tumor stages were evaluated according to the tumor, node and metastasis classification of malignant tumors defined by the American Joint Committee on Cancer (11). All the specimens were directly snap-frozen in liquid nitrogen, and stored at $-130^{\circ} \mathrm{C}$ for the extraction of RNA and total protein. The other parts of the specimens were fixed in buffered formalin for $48 \mathrm{~h}$, embedded in paraffin, and cut into $4 \mu \mathrm{m}$ sections for immunohistochemical detection.

Immumohistochemical staining. Paraffin-embedded samples were cut into $4 \mu \mathrm{m}$ sections and stained with hematoxylin and eosin for tumor confirmation. RACK1 protein expression was visualized using a Streptavidin-Biotin Complex immumohistochemical assay kit (cat. no. SA1027; Wuhan Boster Technology, Ltd., Wuhan, China) according to the manufacturer's protocol. Briefly, the endogenous peroxidase activity of sections was blocked with $\mathrm{H}_{2} \mathrm{O}_{2}$ methanol at room temperature for $10 \mathrm{~min}$ and then incubated in $5 \%$ goat antiserum (Wuhan Boster Biological Technology, Ltd., Wuhan, China) for $15 \mathrm{~min}$ at $37^{\circ} \mathrm{C}$. The sections were then sequentially incubated with a mouse anti-human RACK1 monoclonal antibody (cat. no. sc-17754; dilution, 1:500; Santa Cruz Biotechnology, Inc., Dallas, TX, USA) overnight at $4^{\circ} \mathrm{C}$. The sections were then incubated with biotinylated rabbit anti-mouse immunoglobulin G (cat. no. sc-358914; dilution, 1:2,000; Santa Cruz Biotechnology, Inc.) at $37^{\circ} \mathrm{C}$ for $15 \mathrm{~min}$. A streptavidin-peroxidase complex was added and then 3',3'-diaminobenzidine- $\mathrm{H}_{2} \mathrm{O}_{2}$ was used for the color reaction. Images of four representative fields were captured. The RACK1 positive rate (brown-yellow colored cells) was automatically measured using the Biological Image Analysis System 2000 (Kontron, Eching, Germany).

Immumohistochemical staining was evaluated independently by two pathologists. The level of RACK1 staining was based on the intensity of staining and the proportion of positively stained cancer cells. The following staining scores were applied: Intensity [0 (no staining), 1 (light yellow), 2 (yellow brown), 3 (strong brown color)]; the proportion of positive tumor cells [0 ( $\leq 5 \%$ positive tumor cells), 1 (6-25\% positive tumor cells), 2 (26-50\% positive tumor cells), 3 (51-75\% positive tumor cells) and 4 ( $\geq 76 \%$ positive tumor cells)]. The final basis [immunoreactivity score (IS)] for grouping was the product of the staining area score and staining intensity as follows: 0 , negative; 1-4, weak positive; $5-8$, positive; $9-12$, strong positive.

Reverse transcription-quantitative polymerase chain reaction $(R T-q P C R)$. Total RNA from 8 randomly selected primary tumor and adjacent non-tumor tissue samples were extracted using TRIzol reagent (Invitrogen; Thermo Fisher Scientific, Inc.) according to the manufacturer's protocol. RNA $(2 \mu \mathrm{g})$ from each sample was used for complementary DNA synthesis using the Primescript RT reagent kit (Takara Biotechnology Co., Ltd., Dailan, China). To amplify the spliced form of human RACK1, the following primer sequences were used: forward, 5'-GATTCTGGAAATATTGACTCTT-3' and reverse, 5'-AAC TGGGCCCTTCTGGGTAGAC-3'. GAPDH was used as an internal control and the primer sequences were as follows: forward, 5'-GCACCGTCAAGGCTGAGAAC-3' and reverse,
Table I. Clinical characteristics of respective discovery and verification sets populations.

\begin{tabular}{lc}
\hline Characteristic & Value \\
\hline No. of patients & 157 \\
Sex, male/female & $76 / 81$ \\
Age, years; median, range & $56(35-76)$ \\
CA19-9 level, KU/l; median, range & $131.5(62.1-154.9)$ \\
Tumor size, mm; median, range & $30(29.2-35)$ \\
Pathologic differentiation, & $10 / 31 / 9$ \\
well/moderate/poor & \\
Clinical stage, I/II/III/IV & $7 / 22 / 16 / 5$ \\
\hline
\end{tabular}

CA19-9, cancer antigen 19-9.

5'-TGGTGAAGACGCCAGTGGA-3'. PCR was performed on the ABI prism 7500 sequence detection system (Applied Biosystems; Thermo Fisher Scientific, Inc., USA) and using SYBR Green PCR Master Mix (Applied Biosystems, Thermo Fisher Scientific, Inc., USA), the thermocycler conditions were as follows: $50^{\circ} \mathrm{C}$ for $2 \mathrm{~min}$ and $95^{\circ} \mathrm{C}$ for $10 \mathrm{~min}$, followed by 40 cycles at $95^{\circ} \mathrm{C}$ for $15 \mathrm{sec}$ and $60^{\circ} \mathrm{C}$ for $1 \mathrm{~min}$. The relative expression levels of RACK1 was normalized to that of GAPDH using the comparative $2^{-\Delta \Delta \mathrm{Cq}}$ method (12).

Western blotting. The 8 tumor samples which was selected for RT-qPCR were homogenized in ice-cold radio immunoprecipitation assay lysis buffer at $4^{\circ} \mathrm{C}$ for $15 \mathrm{~min}$ (cat. no. 9806; Cell Signaling Technology, Inc., Danvers, MA, USA) and centrifuged at $13,400 \mathrm{x} \mathrm{g}$ at $4^{\circ} \mathrm{C}$ for $20 \mathrm{~min}$. Then protein concentrations were determined using a Bio-Rad BCA assay kit (Bio-Rad Laboratories, Inc., Hercules, CA, USA). Next, equal amounts of $5 \mathrm{X}$ Laemmli buffer was added and the protein was boiled. $50 \mu \mathrm{g}$ proteins were resolved in $10 \%$ SDS-PAGE and transferred to polyvinylidene fluoride membranes. The membranes were blocked for $1 \mathrm{~h}$ in $5 \%$ bovine milk diluted in TBST at room temperature and incubated with the following antibodies: RACK1 (cat. no. ab72483; 1:500; Abcam, Cambridge, UK) and GAPDH (cat. no. SAB1405848; 1:1,000; Sigma-Aldrich; Merck KGaA, Darmstadt, Germany) at room temperature for 2 h. Following washing with TBST $(0.05 \%$ Tween20) three times, the membranes were subsequently incubated with corresponding horseradish peroxidase-conjugated rabbit anti-mouse immunoglobulin $\mathrm{G}$ (IgG) (cat. no. ab6728; dilution, 1:2,000; Abcam, Cambridge, UK) or goat anti-rabbit IgG (cat. no. ab6721; dilution, 1:2,000; Abcam, Cambridge, UK) for $1 \mathrm{~h}$ at room temperature, and then washed 3 times with TBST. The final band was visualized using an enhanced chemiluminescence assay (Thermo Fisher Scientific, Inc.) and detected using AlphaImager 2200 (ProteinSimple; Bio-Techne, Minneapolis, MN, USA). Statistical analysis was conducted from 3 independent experiments.

Statistical analysis. Statistical analysis was performed using SPSS 18.0 software (SPSS, Inc., Chicago, IL, USA). Results were expressed as the mean \pm standard deviation. Pearson's $\chi^{2}$ test was used to analyze the associations between RACK1 


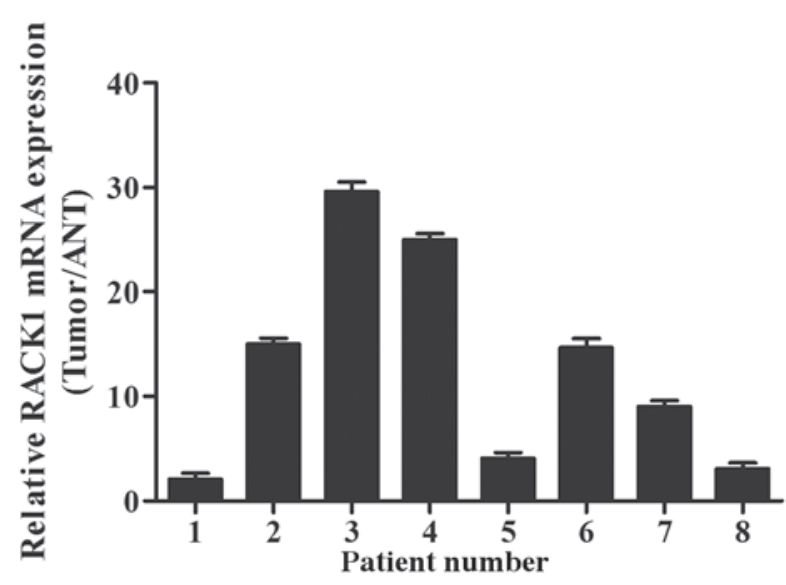

Figure 1. Reverse transcription-quantitative polymerase chain reaction analysis of relative RACK 1 mRNA expression in pancreatic ductal adenocarcinoma tissues and ANT. Data are expressed as the mean \pm standard deviation from three independent experiments. RACK1, receptor for activated $\mathrm{C}$ kinase 1 ; ANT, adjacent noncancerous tissues.

expression and clinicopathological features in patients with PDAC. Univariate and multivariate Cox regression analyses were performed to analyze the survival data. The Kaplan-Meier method and log-rank test were used to evaluate the survival of patients with PDAC.

\section{Results}

RACK1 protein is overexpressed in patients with PDAC. RACK1 mRNA and protein expression levels were assessed by RT-qPCR and western blotting. Of the 8 randomly selected cases, RACK $1 \mathrm{mRNA}$ and protein were overexpressed in 7 out of 8 PDAC tumor specimens compared with in adjacent noncancerous tissues (ANT; Figs. 1 and 2). The medians of RACK1 mRNA and protein expression increased by 2.12 fold and 3.43 fold, respectively (relative ratio of tumor/ANT). In addition, immunohistochemistry confirmed that RACK1 protein was overexpressed in the 121 PDAC lesions compared with their matched adjacent noncancerous tissues (Fig. 3). Furthermore, the IHC data revealed that RACK1 was primarily localized in the cytoplasm of the PDAC cancer cells. No positive staining was identified in the stroma tissues of the tumor (Fig. 3).

RACK1 expression is associated with clinical data in patients with PDAC. The immumohistochemical staining data suggested an increase in the proportion of RACK1 in PDAC tumor tissues compared with adjacent noncancerous tissues. A total of 121/157 (59.2\%) of the PDAC cases exhibited high expression levels of RACK1 (SI $\geq 6)$, whereas 36/157 (40.8\%) had low levels of RACK1 (IS <6). The relationships between RACK1 expression and clinicopathological features are summarized in Table II. The results indicated that RACK1 expression was significantly associated with histological differentiation $(\mathrm{P}<0.001)$, lymph node invasion $(\mathrm{P}<0.001)$ and clinical stage $(\mathrm{P}=0.011)$. However, no associations were observed between RACK1 expression and age, sex, tumor location or tumor size.

Association between RACK1 expression and prognosis in patients with PDAC. To determine whether RACK1

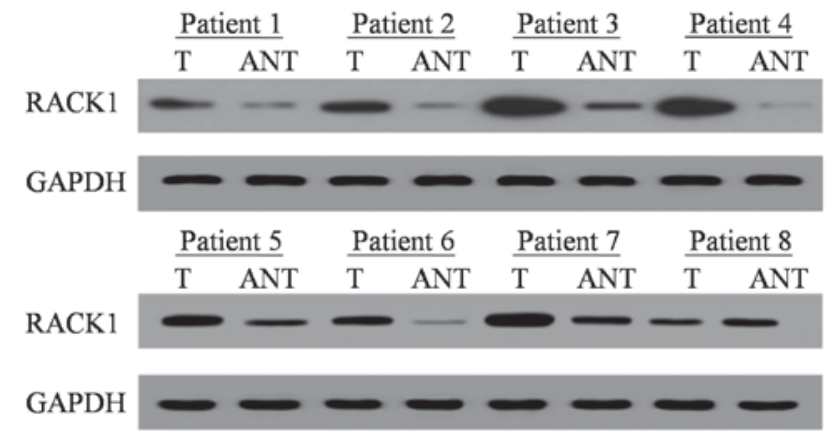

Figure 2. Western blot analysis of RACK1 protein expression in pancreatic ductal adenocarcinoma tissues and ANT. RACK1, receptor for activated $\mathrm{C}$ kinase 1; ANT, adjacent noncancerous tissues; T, tumor tissues.

expression is a prognostic factor for patients with PDAC, Kaplan-Meier analysis and log-rank tests were performed using RACK1 protein expression and clinical follow-up data in all 157 patients. A total of 147 patients died during the follow-up period, and 10 patients were still alive. The results indicated that the median survival time in patients with high RACK1 expression ( $14.3 \pm 1.6$ months; $n=121)$ was significantly shorter compared with in patients with a low RACK1 expression (26.9 \pm 3.2 months; $\mathrm{n}=36 ; \mathrm{P}<0.0001 ;$ Fig. 4A). These results revealed that high level of RACK1 expression indicates poor prognosis for patients with PDAC. Overall survival time was also examined in subgroups of patients. Patients with PDAC in the high RACK1 expression group with early (I and II) and advanced stage (III and IV) PDAC had significantly shorter overall survival times than those in the low RACK1 expression group ( $\mathrm{P}=0.0046$ and $\mathrm{P}<0.001$, respectively; Fig. 4B and $\mathrm{C}$, respectively). In addition, univariate and multivariate analyses revealed that RACK1 expression, histological differentiation, lymph node invasion and tumor resectability were all independent prognostic factors in patients with PDAC (Table III).

\section{Discussion}

Pancreatic ductal adenocarcinoma is known for its poor prognosis (13). The aggressive nature of its invasion, high incidence of early recurrence and poor response to radiotherapy and chemotherapy all contribute to the poor outcomes observed in patients with PDAC (14). To the best of our knowledge, the association between RACK1 expression and prognosis in PDAC was not reported prior to the present study, where RACK1 expression was examined in PDAC in detail.

In the present study, RACK1 expression was revealed to be dramatically higher in PDAC tumor tissues than in adjacent noncancerous tissues. RACK1 expression was associated with T classification, $\mathrm{N}$ classification, clinical stage and liver metastasis, indicating that RACK1 may be a potential biomarker for patients with metastasis. Furthermore, the results of the present study revealed that patients with high RACK1 expression had shorter overall survival times than those with low RACK1 expression. Multivariate analysis revealed that RACK1 expression level was an independent prognostic factor for patients with PDAC. These results suggested that RACK1 was involved in PDAC progression and may be a novel prognostic biomarker for PDAC. 
Table II. Relationship between RACK1 protein expression levels in pancreatic ductal adenocarcinoma and clinical pathological features $(\mathrm{n}=157)$.

RACK1 expression

\begin{tabular}{|c|c|c|c|c|}
\hline \multirow[b]{2}{*}{ Clinical characteristic } & \multirow[b]{2}{*}{$\mathrm{N}(\%)$} & & \multirow[b]{2}{*}{ P-value ${ }^{a}$} \\
\hline & & High $(n=121)$ & Low $(n=36)$ & \\
\hline \multicolumn{5}{|l|}{ Age (years) } \\
\hline$\leq 60$ & 45 (28.7) & $33(73.3)$ & $12(26.7)$ & \multirow[t]{2}{*}{0.213} \\
\hline$>60$ & $112(71.3)$ & $88(78.6)$ & $24(21.4)$ & \\
\hline \multicolumn{5}{|l|}{ Sex } \\
\hline Female & $81(51.6)$ & $64(79.0)$ & $17(21.0)$ & \multirow[t]{2}{*}{0.106} \\
\hline Male & $76(48.4)$ & $57(75.0)$ & $11(25.0)$ & \\
\hline \multicolumn{5}{|l|}{ Tumor location } \\
\hline Head & $123(78.3)$ & $100(81.3)$ & $23(18.7)$ & \multirow[t]{2}{*}{0.165} \\
\hline Body/tail & $34(21.7)$ & $21(61.8)$ & $13(38.2)$ & \\
\hline \multicolumn{5}{|c|}{ Histological differentiation } \\
\hline Well & $22(14)$ & $12(54.5)$ & $10(45.5)$ & \multirow[t]{2}{*}{$<0.001$} \\
\hline Moderate/poor & $135(86)$ & $109(80.7)$ & $26(19.3)$ & \\
\hline \multicolumn{5}{|l|}{ Tumor size } \\
\hline$\leq 2 \mathrm{~cm}$ & $62(39.5)$ & $43(69.4)$ & $19(30.6)$ & \multirow[t]{2}{*}{0.123} \\
\hline$>2 \mathrm{~cm}$ & $95(60.5)$ & $79(83.2)$ & $16(16.8)$ & \\
\hline \multicolumn{5}{|l|}{ Lymph node invasion } \\
\hline Absent & $32(20.4)$ & $20(62.5)$ & $12(37.5)$ & \multirow[t]{2}{*}{$<0.001$} \\
\hline Present & $125(79.6)$ & $101(80.8)$ & $24(19.2)$ & \\
\hline \multicolumn{5}{|l|}{ Clinical stage } \\
\hline $\mathrm{I}+\mathrm{II}$ & $87(55.4)$ & $66(75.9)$ & $21(24.1)$ & \multirow[t]{2}{*}{0.011} \\
\hline III+IV & $70(44.6)$ & $55(78.6)$ & $15(21.4)$ & \\
\hline
\end{tabular}

apearson's $\chi^{2}$ test. RACK1, receptor for activated C kinase 1.
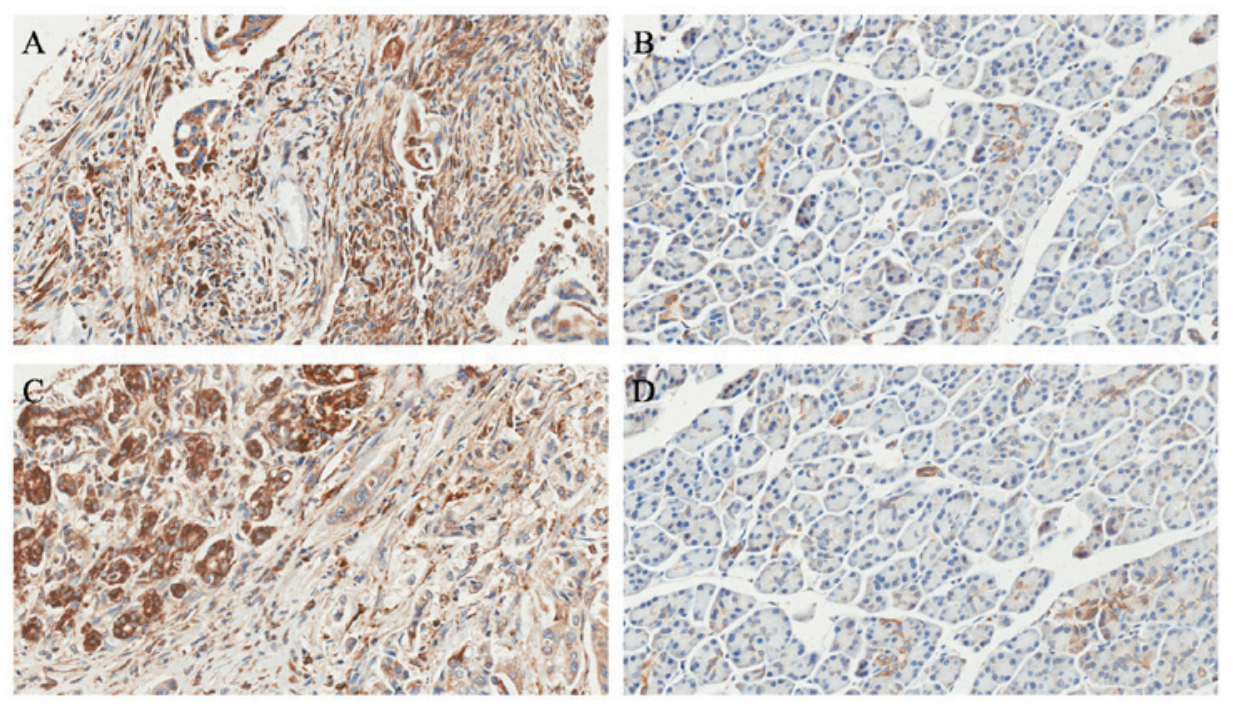

Figure 3. Immumohistochemical staining of receptor for activated C kinase 1 in (A and C) pancreatic ductal adenocarcinoma tissues and (B and D) adjacent noncancerous tissues (magnification, $\mathrm{x} 200$ ).

Several novel prognostic factors including C-C motif chemokine ligand 18 (15), golgi phosphoprotein 3 (16), and UL16 binding protein 2 (17) have previously been reported. In addition, tumor grade, lymph node invasion and clinical stage have also been demonstrated to be correlated with outcome in patients with PDAC (18-20). However, the efficacy of such prognostic markers in clinical application remains unknown. Therefore, further investigation is warranted to discover novel 
Table III. Univariate and multivariate analysis of prognostic parameters for survival in patients with pancreatic ductal adenocarcinoma.

\begin{tabular}{|c|c|c|c|c|c|c|}
\hline \multirow[b]{2}{*}{ Parameter } & \multicolumn{3}{|c|}{ Univariate analysis } & \multicolumn{3}{|c|}{ Multivariate analysis } \\
\hline & RR & $95 \% \mathrm{CI}$ & P-value & RR & $95 \% \mathrm{CI}$ & P-value \\
\hline Expression of RACK1, high vs. low & 1.901 & $1.141-3.012$ & 0.006 & 2.712 & $1.566-4.691$ & 0.002 \\
\hline Age,$\leq 60$ vs. $>60$ & 1.211 & $0.801-1.901$ & 0.812 & 1.354 & $0.866-2.102$ & 0.223 \\
\hline Sex, male vs. female & 0.999 & $0.678-1.499$ & 0.924 & 1.112 & $0.647-1.623$ & 0.857 \\
\hline Tumor location, head vs. body/tail & 0.821 & $0.465-1.436$ & 0.554 & 0.643 & $0.379-1.242$ & 0.124 \\
\hline $\begin{array}{l}\text { Histological differentiation, } \\
\text { well vs. moderate/poor }\end{array}$ & 1.613 & $0.876-3.512$ & 0.001 & 1.721 & $0.976-2.993$ & 0.005 \\
\hline Size,$\leq 2 \mathrm{~cm}$ vs. $>2 \mathrm{~cm}$ & 1.966 & $1.132-3.499$ & 0.011 & 1.666 & $0.899-2.902$ & 0.219 \\
\hline Lymph node invasion, absent vs. present & 1.112 & $0.743-1.699$ & 0.222 & 0.623 & $0.399-0.976$ & 0.023 \\
\hline Liver metastasis, absent vs. present & 1.342 & $0.787-2.243$ & 0.325 & 1.032 & $0.387-3.532$ & 0.933 \\
\hline Clinical stage, I vs. II vs. III vs. IV & 1.376 & $1.154-1.599$ & 0.021 & 1.498 & $0.731-2.675$ & 0.221 \\
\hline Treatment, radical vs. palliative & 2.499 & $1.632-3.812$ & 0.001 & 2.932 & $1.790-4.659$ & 0.001 \\
\hline
\end{tabular}

RACK1, receptor for activated C kinase 1 ; RR, relative risk; CI, confidence interval.

\section{A}

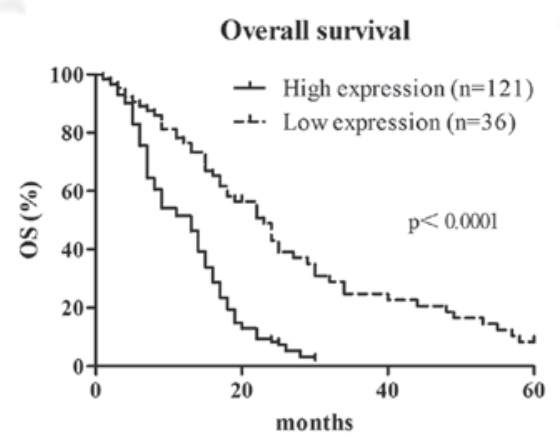

C

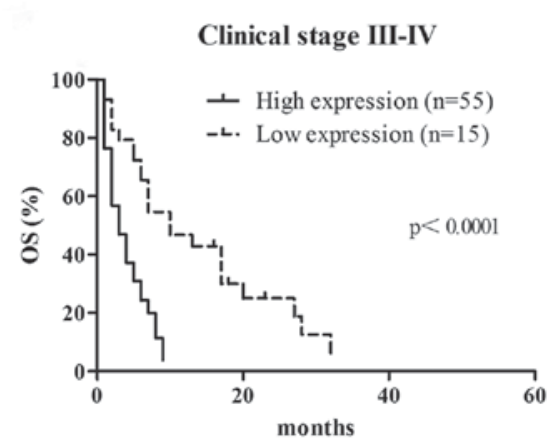

B

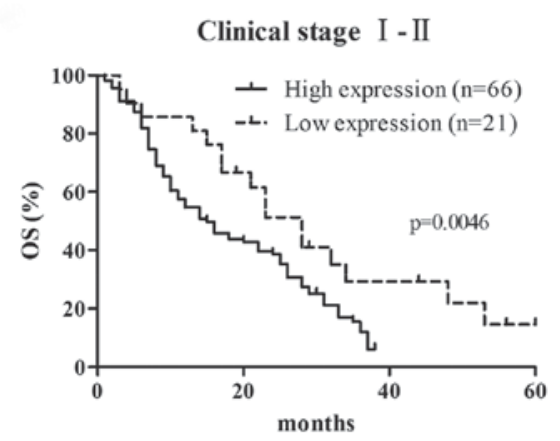

Figure 4. Kaplan-Meier curves of survival differences among patients with PDAC with (A) high RACK1 expression (n=121) and low RACK1 expression $(\mathrm{n}=36)$, and patients with PDAC in the high and low RACK1 expression groups with (B) early (I and II) and (C) advanced stage (III and IV) PDAC. P-values were determined using the log-rank test. PDAC, pancreatic ductal adenocarcinoma; RACK1, receptor for activated C kinase 1; OS, overall survival.

prognostic markers with improved prognostic efficacy in patients with PDAC.

RACK1, which is upregulated in several solid malignancies, was originally reported in colorectal cancer (21). RACK1 serves as a scaffold and anchoring protein for PKC, and stabilizes the activated conformation of PKC. RACK1 has been associated with several signaling pathways, and serves a vital function in tumorigenesis (22). RACK1 was reported to be overexpressed in multiple cancers including melanoma (23), gastric cancer (24), hepatocellular carcinoma (25) and pulmonary adenocarcinoma (26). RACK1 is thought to promote tumor invasion and metastasis. However, RACK1 also suppresses tumor growth in gastric cancer, indicating that RACK1 may serve different functions in different tumors. 
Mamidipudi and Cartwright (27) reported that RACK1 inhibited colorectal cancer cell proliferation via the regulation of Src activity. However, Saito et al (28) reported that RACK1 was overexpressed in colon cancer, and promoted colon cancer cell proliferation. Thus, the function of RACK1 varies between tumor types, and remains to be elucidated. The present study measured RACK1 mRNA and protein expression levels in tumor tissues and adjacent noncancerous tissues from 157 patients with PDAC. The results revealed that RACK1 was overexpressed in tumor tissues compared with adjacent noncancerous tissues. RACK1 was associated with clinical stage, differentiation and lymph node metastasis in patients with PDAC.

In conclusion, RACK1 was overexpressed in patients with PDAC, and positively associated with the degree of malignancy in patients with PDAC. Therefore, RACK1 may serve as a novel biomarker and potential therapeutic target. Further investigations, in particular those concerning the molecular mechanisms underlying the effect of RACK1 in PDAC, are being performed.

\section{References}

1. Buekki J: Pancreatic adenocarcinoma. N Engl J Med 371: 2139-2140, 2014

2. Iacobuzio-Donahue CA and Herman JM: Autophagy, p53, and pancreatic cancer. N Engl J Med 370: 1352-1353, 2014.

3. Viale A, Pettazzoni P, Lyssiotis CA, Ying H, Sanchez N, Marchesini M, Carugo A, Green T, Seth S, Giuliani V, et al: Oncogene ablation-resistant pancreatic cancer cells depend on mitochondrial function. Nature 514: 628-632, 2014.

4. Meacham CE and Morrison SJ: Tumour heterogeneity and cancer cell plasticity. Nature 501: 328-337, 2013.

5. Jin S, Mu Y, Wang X, Liu Z, Wan L, Xiong Y, Zhang Y, Zhou L and Li L: Overexpressed RACK1 is positively correlated with malignant degree of human colorectal carcinoma. Mol Biol Rep 41: 3393-3399, 2014.

6. Hu L, Lu F, Wang Y, Liu Y, Liu D, Jiang Z, Wan C, Zhu B, Gan L, Wang Y, et al: RACK1, a novel hPER1-interacting protein. J Mol Neurosci 29: 55-63, 2006.

7. Cho IR, Kaowinn S, Moon J, Soh J, Kang HY, Jung CR, Oh S, Song H, Koh SS and Chung YH: Oncotropic H-1 parvovirus infection degrades HIF-1alpha protein in human pancreatic cancer cells independently of VHL and RACK1. Int J Oncol 46: 2076-2082, 2015.

8. Sato T, Takahashi H, Hatakeyama S, Iguchi A and Ariga T: The TRIM-FLMN protein TRIM45 directly interacts with RACK1 and negatively regulates PKC-mediated signaling pathway. Oncogene 34: 1280-1291, 2015.

9. Cheng D, Qian W, Wang Y, Meng M, Wei L, Li Z, Kang L, Peng $\mathbf{J}$ and $\mathrm{Xia}$ Q: Nuclear import of transcription factor BR-C is mediated by its interaction with RACK1. PloS One 9: e109111, 2014.

10. Omosigho NN, Swaminathan K, Plomann M, Mueller-Taubenberger A, Noegel AA and Riyahi TY: The Dictyostelium discoideum RACK1 orthologue has roles in growth and development. Cell Commun Signal 12: 37, 2014.

11. Edge SB and Compton CC: The American Joint Committee on Cancer: the 7th edition of the AJCC cancer staging manual and the future of TNM. Ann Surg Oncol 17: 1471-1474, 2010.
12. Livak KJ and Schmittgen TD: Analysis of relative gene expression data using real-time quantitative PCR and the 2(-Delta Delta C(T)) method. Methods 25: 402-408, 2001.

13. Waddell N, Pajic M, Patch AM, Chang DK, Kassahn KS, Bailey P, Johns AL, Miller D, Nones K, Quek K, et al: Whole genomes redefine the mutational landscape of pancreatic cancer. Nature 518: 495-501, 2015.

14. Junttila MR and de Sauvage FJ: Influence of tumour micro-environment heterogeneity on therapeutic response. Nature 501: 346-354, 2013.

15. Meng F, Li W, Li C, Gao Z, Guo K and Song S: CCL18 promotes epithelial-mesenchymal transition, invasion and migration of pancreatic cancer cells in pancreatic ductal adenocarcinoma. Int J Oncol 46: 1109-1120, 2015.

16. Zhang LJ, Wang KB, Liu LS, Chen LZ, Peng BG, Liang LJ, Li Z, Xue L, Li W and Xia JT: Overexpression of GOLPH3 is associated with poor prognosis and clinical progression in pancreatic ductal adenocarcinoma. BMC Cancer 14: 571, 2014.

17. Chang YT, Wu CC, Shyr YM, Chen TC, Hwang TL, Yeh TS, Chang KP, Liu HP, Liu YL, Tsai MH, et al: Secretome-based identification of ULBP2 as a novel serum marker for pancreatic cancer detection. PloS One 6: e20029, 2011.

18. Oron Y: Integrin-based therapy of pancreatic adenocarcinoma: Current status and future perspectives. Minerva Gastroenterol Dietol 61: 71-86, 2015.

19. Strobel O, Hinz U, Gluth A, Hank T, Hackert T, Bergmann F, Werner J and Buchler MW: Pancreatic adenocarcinoma: Number of positive nodes allows to distinguish several $\mathrm{N}$ categories. Ann Surg 261: 961-969, 2015.

20. Bever KM, Sugar EA, Bigelow E, Sharma R, Laheru D, Wolfgang CL, Jaffee EM, Anders RA, De Jesus-Acosta A and Zheng L: The prognostic value of stroma in pancreatic cancer in patients receiving adjuvant therapy. HPB (Oxford) 17: 292-298, 2015.

21. Cui J, Chen Y, Wang HY and Wang RF: Mechanisms and pathways of innate immune activation and regulation in health and cancer. Hum Vaccin Immunother 10: 3270-3285, 2014.

22. Myklebust LM, Horvli O and Raae AJ: RACK1 (receptor for activated C-kinase 1) interactions with spectrin repeat elements. J Mol Recognit 28: 49-58, 2015.

23. Campagne C, Jule S, Alleaume C, Bernex F, Ezagal J, Chateau-Joubert S, Estrada M, Aubin-Houzelstein G, Panthier JJ and Egidy G: Canine melanoma diagnosis: RACKI as a potential biological marker. Vet Pathol 50: 1083-1090, 2013.

24. Deng YZ, Yao F, Li JJ, Mao ZF, Hu PT, Long LY, Li G, Ji XD, Shi S, Guan DX, et al: RACK1 suppresses gastric tumorigenesis by stabilizing the beta-Catenin destruction complex. Gastroenterology 142: 812.e15-823.e15, 2012.

25. Ruan Y and Gu J: O-GlcNAc modification of ribosomal RACK1 enhances hypoxia-induced epithelial-mesenchymal transition in hepatocellular carcinoma. Glycobiology 23: 1345-1345, 2013.

26. Choi YY, Lee SY, Lee WK, Jeon HS, Lee EB, Lee HC, Choi JE, Kang HG, Lee EJ, Bae EY, et al: RACK1 is a candidate gene associated with the prognosis of patients with early stage non-small cell lung cancer. Oncotarget 6: 4451-4466, 2015.

27. Mamidipudi V and Cartwright CA: A novel pro-apoptotic function of RACK1: Suppression of Src activity in the intrinsic and Akt pathways. Oncogene 28: 4421-4433, 2009.

28. Saito A, Fujii G, Sato Y, Gotoh M, Sakamoto M, Toda G and Hirohashi S: Detection of genes expressed in primary colon cancers by in situ hybridisation: Overexpression of RACK 1 . Mol Pathol 55: 34-39, 2002.

This work is licensed under a Creative Commons Attribution-NonCommercial-NoDerivatives 4.0 International (CC BY-NC-ND 4.0) License. 